\title{
Philosophiques
}

\section{Peter Kemp (dir.), Le discours bioéthique, Paris, Cerf, coll. " Recherches morales ", 2004, 113 pages.}

\section{Dominic Desroches}

Volume 32, numéro 2, automne 2005

URI : https://id.erudit.org/iderudit/011881ar

DOI : https://doi.org/10.7202/011881ar

Aller au sommaire du numéro

Éditeur(s)

Société de philosophie du Québec

ISSN

0316-2923 (imprimé)

1492-1391 (numérique)

Découvrir la revue

Citer ce compte rendu

Desroches, D. (2005). Compte rendu de [Peter Kemp (dir.), Le discours bioéthique, Paris, Cerf, coll. « Recherches morales », 2004, 113 pages.] Philosophiques, 32(2), 462-465. https://doi.org/10.7202/011881ar d'utilisation que vous pouvez consulter en ligne.

https://apropos.erudit.org/fr/usagers/politique-dutilisation/ 
du PP. Ainsi, l'auteur, commentant son propre exemple, affirme qu'une des conséquences de l'action envisagée (diminution de la fertilité humaine) est peu plausible: «cette conséquence a une probabilité extrêmement faible» (p. 184). Mais parler ainsi, c'est accorder aux partisans orthodoxes du PP que l'on est dans le domaine de la prévention et non dans celui de la précaution. Pour eux, en effet, un risque non avéré, relevant de la précaution, n'est pas un risque extrêmement peu probable, mais un risque dont la probabilité ne peut pas être déterminée 8 .

Au total, M. H. a voulu montrer que les biotechnologies, dans leurs avancées les plus radicales, sont l'occasion d'effectuer un travail «d'explicitation herméneutique à des fins normatives» (p. 126). Ce travail est mené avec une remarquable détermination. Mais bien des choses sont encore à préciser, à commencer par le concept de «contexte moral objectif ». M. H. a recours à toute une série de métaphores pour le caractériser; il en parle à l'occasion comme d'un agent intentionnel, acceptant certaines pratiques et refusant d'en admettre d'autres: cette stratégie n'aide pas réellement le lecteur à y voir clair. En outre, les sociétés au sein desquelles les biotechnologies ont les effets les plus spectaculaires sont aussi des sociétés multiculturelles. Quelle place accorder au discours des dissidents dans le contexte moral objectif? Comment distinguer ceux qui s'affranchissent du CMO et ceux qui le réforment en profondeur? Ce sont là des questions qui restent ouvertes.

JEAN-YVES GOFFI

Université de Grenoble

\section{Peter Kemp (dir.), Le discours bioéthique, Paris, Cerf, coll. «Recherches morales », 2004, 113 pages.}

Les lecteurs intéressés par les questions difficiles se trouvant au carrefour de certaines disciplines des sciences sociales, notamment la bioéthique, l'éthique et le droit, seront heureux d'apprendre la publication en langue française de ce petit livre consacré aux rapports nouveaux qui unissent désormais la bioéthique et le biodroit. Sous le titre général Le discours bioéthique, les textes réunis ici ont tout d'abord été prononcés en anglais à titre de communications lors de deux colloques organisés par le Centre de recherche en éthique et droit dans la nature et la société (Center for Etik og Ret), à Copenhague, en 1996 et en 1998. Ce ne sont donc pas des textes neufs, mais des communications revues et augmentées en vue de leur publication pour le lectorat francophone. Ces recherches, qui n'ont de bioéthique que le thème, sont d'une très grande actualité et traduisent toute l'importance que les problèmes d'éthique, précisément ceux que l'on rencontre en santé, occuperont sous peu pour les sciences juridiques. Sans tarder, voyons ce que peuvent nous apprendre ces recherches qui portent sur le développement actuel du "discours bioéthique».

Le premier texte, qui donne le ton du livre, est de Peter Kemp. Le directeur du Centre de recherche en éthique et droit, bien connu pour ses nombreux travaux en philosophie française, en éthique et en bioéthique, propose une réflexion générale ayant pour but de situer l'ouvrage. Ainsi, il se penche sur les rapports complexes

8. C'est la différence entre prendre une décision en situation de risque et prendre une décision en situation d'incertitude. 
qu'entretiennent les domaines de la bioéthique et du biodroit. Après avoir rappelé de manière générale la problématique et les concepts-clés de ces nouveaux "savoirs ", Kemp, qui s'intéresse surtout à l'introduction du biodroit dans l'éthique, situe le terme "biodroit » à l'intérieur de la tradition juridique française. Or ce premier constat vise à établir la possibilité de son dépassement. Car en prenant l'exemple du Danemark, un petit pays où la définition du biodroit jouit d'une assise philosophique, Kemp veut dépasser une bioéthique qui se conçoit, trop souvent, certes, sur le modèle désuet du Common law et le biodroit qui, lui, se conçoit de manière hiérarchique, en France notamment. Comme on le voit, cette première analyse de Kemp a donc pour mission de présenter les liens unissant la bioéthique et le droit, tout en tentant de comprendre en même temps la place récente qu'occupe le biodroit en éthique. Cette manière, disons circulaire, de concevoir ces nouvelles disciplines permet à Kemp de rappeler que les rapports entre la bioéthique et le biodroit ne relèvent pas simplement de sphères isolées, mais plutôt d'influences réciproques à la faveur desquelles ces domaines se limitent l'un l'autre (33).

La présentation suivante est du philosophe Paul Ricoeur. Son étude met précisément l'accent sur le jugement dans l'orientation thérapeutique (ou clinique) de la bioéthique. Ricoeur s'intéresse ici de manière générale au jugement et à ses applications dans le large domaine de la santé. Ainsi propose-t-il de distinguer d'abord le jugement prudentiel du jugement déontologique et du jugement réflexif. S’inspirant de la tradition philosophique (on pense particulièrement à Aristote), Ricoeur précisera la nature du jugement dans l'éthique médicale en insistant sur le fait que ce qui est en jeu, c'est la sagesse, c'est-à-dire la qualité du jugement que l'on doit retrouver dans une situation qui exige l'application de règles universelles à l'intérieur d'une pratique toujours singulière. En partant du jugement prudentiel (phronésis), général en ce qu'il concerne toutes les situations de la vie, Ricoeur explicite le passage au niveau déontologique, précisément lorsque le jugement doit s'appliquer à une profession. L'auteur justifie ce passage au moyen de trois fonctions du jugement déontologique; celui-ci doit: 1) universaliser les préceptes relevant du pacte entre le malade et le médecin; 2) assurer une connexion entre les normes; 3 ) arbitrer, suivant son histoire, les conflits à l'intérieur de la profession (40-46). Cela étant compris, Ricoeur montre finalement comment le jugement devra s'accomplir dans la réflexion propre à l'éthique médicale. L'auteur, qui met beaucoup l'accent sur les thèmes de la dignité, de la singularité et de la souffrance, terminera son texte par deux remarques qui visent à rappeler que le jugement (qu'il avait auparavant abordé et développé dans «Petite éthique dans Soi même comme un autre) s'inscrit toujours dans un monde «humain » qui comprend bien entendu l'espace de la clinique médicale où se vit toujours la souffrance et la fragilité.

Le texte suivant est de la philosophe Anne Fagot-Largeault. Bien connue pour ses travaux en éthique et en bioéthique, celle-ci s'interroge sur le fondement des bionormes. Elle se pose une question à laquelle elle n'entend pas donner une réponse dernière, mais seulement proposer quelques pistes de réflexion générales. Le problème se formule tout simplement en ces termes: les bionormes se trouvent-elles dans la nature biologique ou dans la rationalité humaine? (51) En partant du principe que l'homme modifie toujours davantage son environnement, l'auteure s'interrogera d'abord elle-même, tout en posant aussi quelques questions à son lecteur, sur la meilleure façon de cerner le lieu des bionormes. Elle discutera, en s'appuyant notamment sur les idées des philosophes Kant, Spinoza, Bergson et Jonas, pour ne pas les 
nommer, quelques cas où les bionomes semblent se trouver dans la nature. Or, remarquera-t-elle fort justement, si l'on trouve des critères fondés en nature, l'évaluation que l'on fait des normes repose toujours, en dernière analyse, sur le jugement humain. La réflexion audacieuse menée par Fagot-Largeault, qui rejoint ici celle de Ricoeur, s'achève sur une double question: 1) La nature doit-elle être remodelée par les humains?; et 2) Y a-t-il un sens absolu au bien inscrit dans la nature? Sans pouvoir donner une réponse définitive à ces deux questions - sont-elles bien posées et méritentelles des réponses définitives, demanderons-nous? - il ressort de cette réflexion courageuse sur les bionormes que la raison humaine tend vers la "vie bonne ", mais que notre nature, cependant, finie et obsédée par le progrès, peut toujours accomplir le monstrueux. La fabrication du monstrueux, lorsqu'on vise à faire le bien, conclut notre auteure, demeure une perspective pour le moins troublante (67-68).

Evandro Agazzi entend pour sa part réfléchir sur le concept de «nature » dans le discours éthique. Il se demande en effet de quelle nature il s'agit lorsque l'on fait appel à ce terme en éthique. Autrement dit, que présuppose aujourd'hui l'idée de nature pour nous, hommes? Après avoir distingué la morale de l'éthique - il faut ici remercier Agazzi de cette distinction que les professionnels de la bioéthique, jusqu'à preuve du contraire, demeurent incapables de faire - et souligné les caractéristiques de l'éthique traditionnelle (74), l'auteur aborde les questions d'éthique dans le contexte de la sécularisation. Sa manière de concevoir l'éthique l'aide à repenser l'idée de responsabilité qui appelle toujours, selon lui, le respect. Comment parvient-il à cette liaison nécessaire entre la responsabilité et le respect? Inspiré sans aucun doute par Aristote, qu'il ne cite pas, l'auteur veut voir une hiérarchie ontologique dans la nature, au nom de laquelle les plantes restent inférieures aux animaux et les animaux inférieurs aux hommes. Mais ici, Agazzi entend se dégager de la perspective anthropocentriste que sa pensée lui suggère pourtant; loin de placer l'homme au centre du monde de manière arbitraire, Agazzi préfère se dire cosmocentriste, en insistant par exemple sur le fait que l'être humain ne doit pas faire ce qu'il veut des autres êtres, bien qu'il ait des droits supérieurs. Agazzi veut que l'être humain agisse de façon responsable. L'argumentaire d'Agazzi essaie tant bien que mal de se forger une voie entre la théorie d'Aristote et celle de Singer. Cela, on s'en doute, n'est pas très facile. Reprenant finalement à nouveaux frais la distinction classique entre l'artificiel et le naturel - distinction qui n'exclut plus, aux yeux d'Agazzi, la possibilité pour l'homme de modifier la nature, car c'est essentiellement dans la nature de l'homme que de modifier la nature... - notre auteur est conduit, au nom de l'ontologie axiologique à laquelle il se rattache, à défendre la nature en vertu de la notion éthique de respect. Cette notion de respect, reprise à Kant (78-79), consistera en gros à trouver le juste milieu entre le droit de l'homme à satisfaire ses besoins et le souci que l'on doit attribuer à tous les êtres naturels selon leur dignité ontologique (85).

Or Marco Olivetti ne peut partager les idées de son collègue. Car si Agazzi comprend la responsabilité avec la notion de réciprocité, Olivetti le refuse. Dans sa communication, selon son titre du moins, consacrée au passage des droits aux biodroits, dont il ne parlera pas en tant que tels, Olivetti soutiendra la thèse selon laquelle les autres êtres vivants ont des droits parce que la vie est une finalité en elle-même. Cherchant du secours chez les philosophes (Descartes, Hume, Kant et Heidegger), Olivetti, qui s'imagine bien que les droits présupposent un agent pour les respecter, tient cependant mordicus à défendre l'idée d'un droit conçu de manière asymétrique. L'argument principal en faveur de sa thèse est que les droits humains ne sont pas 
supérieurs aux autres droits (aux droits des végétaux ou des animaux par exemple) puisqu'à l'origine, la dignité humaine ne coïncidait pas avec les droits humains. La supériorité de ces derniers n'a, en fait, rien d'intrinsèque (86-87). Dans le contexte de la fondation des biodroits à partir de l'éthique qui est désormais le nôtre, Olivetti est conduit à son tour à soutenir que la responsabilité repose fondamentalement sur la comparaison des droits, y compris des droits des non-vivants. Le droit qui l'emportera sera finalement celui qui favorise ma responsabilité à l'égard de l'autre être, envers qui j'ai un devoir en tant que fin en soi (98). Curieusement, donc, la responsabilité éthique ne reposera pas, selon Olivetti, sur ma conscience morale ou sur un sentiment quelconque envers les autres vivants, mais plutôt sur une froide comparaison (utilitariste?) entre des droits...

Dans les dernières pages, Kemp assurera lui-même, au nom du centre de recherche en éthique et droit qu'il dirige, la présentation de quatre principes éthiques essentiels pour bien comprendre le développement du discours bioéthique. Il s'agit des principes de l'autonomie, de la dignité, de l'intégrité et de la vulnérabilité, ce dernier principe trouvant en Kemp, disciple officiel de Ricoeur et de Lévinas, un grand défenseur. Or Kemp insiste sur un point qui nous paraît très important. Pour saisir leur portée, en effet, il ne faut jamais concevoir ces quatre principes éthiques indépendamment les uns des autres puisqu'ils doivent, dans l'évaluation éthique, toujours travailler ensemble. Autrement dit, ces quatre principes éthiques n'auront un sens "éthique» que s'ils sont reconnus dialectiquement ou organiquement, c'est-à-dire s'ils sont rattachés ensemble. Dès lors, si l'on ne veut pas manquer l'éthique - qui est au cœur des questions de bioéthique -, il faut éviter d'isoler, pour une raison quelconque, l'un des quatre principes. Entendus et développés «co-originairement », pour reprendre un terme qu'affectionnait le jeune Heidegger, ces principes aideront à mieux voir ce qu'est l'éthique, c'est-à-dire la recherche de la vie bonne.

Ce florilège de textes sera apprécié par les personnes qui veulent s'initier aux questions de biodroit rattachées à la bioéthique, donc au premier chef les étudiants du premier cycle universitaire inscrits en bioéthique et en droit. Les auteurs proposent des repères utiles pour comprendre le passage de l'éthique à la bioéthique et la montée récente du biodroit. Si la valeur des textes est inégale, les sujets abordés restent, en revanche, d'une grande actualité. Ces petites contributions, qui ne refusent jamais les détours qu'implique la réflexion philosophique (celle qui fonde bien entendu l'éthique), sont toutes traversées par un même fil conducteur: elles suggèrent de replacer au centre du discours bioéthique la notion de respect, aujourd'hui tombée dans l'oubli. $\mathrm{Ne}$ serait-ce que pour cette raison, nous recommanderons vivement la lecture de ce petit livre.

DOMINIC DESROCHES

Université de Montréal

\section{Thomas De Koninck, Philosophie de l'éducation. Essai sur le devenir humain, Paris, Presses Universitaires de France, coll. «Thémis», 2004, 296 pages.}

Philosophie de l'éducation comporte sept chapitres (L'enfance, Le refus de la violence et le pouvoir de la beauté, Les lettres vivantes, Le politique, L'enseignement et les savoirs, La médecine et l'éthique, Les questions ultimes) et est un recueil de méditations 